\title{
MÉTODO PARA A DETERMINAÇÃO DE HEXAZINONE E TEBUTIURON EM ÁGUA
}

\author{
Vera L. Ferracini, Sonia C. N. Queiroz*, Marco A. F. Gomes e Gustavo L. Santos \\ Embrapa Meio Ambiente, CP 69, 13820-000 Jaguariúna - SP
}

Recebido em 16/12/03; aceito em 4/1/05; publicado na web em 28/2/05

\begin{abstract}
METHOD FOR DETERMINATION OF HEXAZINONE AND TEBUTHIURON IN WATER. This work presents an alternative method for determination of the herbicides tebuthiuron and hexazinone in ground water. The extraction was made with dichloromethane and the analyses by high performance liquid chromatography (HPLC), using reversed-phase column, C-18, mobile phase methanol/water 50:50, v/v, detection and quantification at $247 \mathrm{~nm}$. The following validation parameters were obtained: limit of detection of method 0.02 and $0.03 \mu \mathrm{g} \mathrm{L}{ }^{-1}$, limit of quantification of method 0.07 and $0.09 \mu \mathrm{g} \mathrm{L}^{-1}$; linear range limit of quantification of instrument $-300 \mu \mathrm{g} \mathrm{L}^{-1}\left(\mathrm{r}^{2} \geq 0.998\right)$; recoveries from 90.3 to $108.2 \%$ and 90.3 to $101.6 \%$; intermediary precision $(\% \mathrm{RSD})<8$ and $<6 \%$, for hexazinone and tebuthiuron, respectively. The method showed to be efficient and reliable for determination of the herbicides in ground water.
\end{abstract}

Keywords: herbicides; high performance liquid chromatography; ground water.

\section{INTRODUÇÃO}

Os sistemas de produção intensivos elevam a necessidade de uso de agroquímicos, os quais aumentam os níveis de nitrato, fosfato e as concentrações residuais dos agrotóxicos, ou de seus metabólitos, que, por sua vez, podem comprometer a qualidade das águas superficiais e subterrâneas. A deriva de agrotóxicos, ocorrida durante o processo de aplicação dos produtos, diminui a eficiência da aplicação, além de comprometer a qualidade da flora e da fauna nativas, assim como da água local e de outras regiões, sem falar da própria saúde do trabalhador rural e de comunidades vizinhas.

Os agrotóxicos possuem diferentes estruturas e atividades biológicas, que os tornam bastante diferenciados quanto a seus efeitos de ordem fitossanitária. Estes, aliás, são os principais motivos pelos quais são amplamente utilizados na agricultura. Devido ao fato de serem potencialmente tóxicos aos organismos, o destino destes produtos no ambiente deve ser investigado ${ }^{1}$.

As áreas de recarga direta ou de afloramento do Aqüífero Guarani têm se mostrado bastante expostas ao risco de degradação, seja pelo uso intenso de agrotóxicos seja por processos erosivos, principalmente devido a dois fatores fundamentais: a) alta vulnerabilidade natural das mesmas e b) avanço das atividades agrícolas sobre elas, sem critério em relação à aptidão ou mesmo de capacidade de uso de seus solos.

Os herbicidas hexazinone (classe das triazinonas) e o tebutiuron (classe das fenilureas), dentre outros, vêm sendo aplicados intensivamente no cultivo de cana-de-açúcar, na região de Ribeirão Preto. Na microbacia do Córrego Espraiado, localizado nesta região, encontra-se um dos pontos de recarga do aqüífero Guarani, que são áreas susceptíveis à contaminação por agrotóxicos devido à monocultura da cana-de-açúcar e à presença de solo arenoso ${ }^{2,3}$. Conforme citado na literatura, o hexazinone e o tebutiuron (Figura 1) possuem alto potencial de lixiviação e, conseqüentemente, podem atingir as águas subterrâneas ${ }^{4}$. Assim, há necessidade de se realizar um monitoramento da qualidade da água oriunda deste local, para garantir a saúde da população. Para isso torna-se necessária a

*e-mail: sonia@cnpma.embrapa.br disponibilização de metodologias rápidas e eficientes para a detecção e quantificação destas espécies.

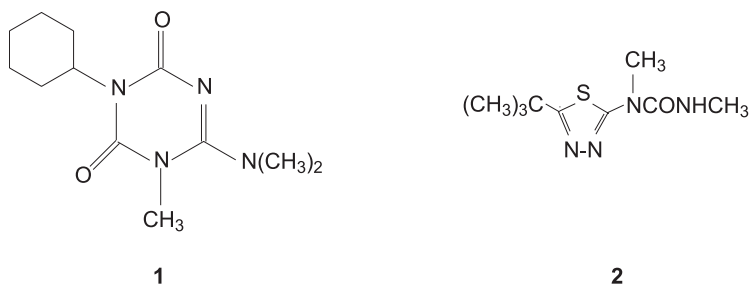

Figura 1. Estruturas dos herbicidas: 1) hexazinone e 2) tebutiuron

Existem vários métodos na literatura descrevendo técnicas para a determinação de hexazinone e seus metabólitos em várias matrizes, incluindo eletroforese capilar ${ }^{5}$, cromatografia gasosa com detector seletivo para nitrogênio ${ }^{6}$, cromatografia líquida com detector de UV/Vis ${ }^{7}$ e acoplada a espectrometria de massas ${ }^{8}$. Um método para a determinação multirresíduos de agrotóxicos, baseado em extração em fase sólida seguido de CLAE, foi proposto por Queiroz e colaboradores ${ }^{9}$, entretanto o método inclui apenas o tebutiuron.

Assim, o presente trabalho apresenta um método, baseado em extração líquido-líquido seguida de CLAE, para a determinação simultânea de hexazinone e tebutiuron em amostras de água.

\section{PARTE EXPERIMENTAL}

\section{Reagentes e solventes}

Os padrões de agrotóxicos foram tebutiuron $(99,5 \%)$ adquirido da firma Dr. Ehrenstorfer e hexazinone (99,9\%) doado pela DuPont. O sulfato de sódio anidro, p.a. (99\%), foi obtido da Ecibra.

Os solventes utilizados foram diclorometano, grau resíduos $(99,9 \%)$ da Tedia; acetona, grau P.A., da Allkimia e metanol, grau cromatográfico, da Mallinckrodt Ultimar.

\section{Preparo das soluções analíticas}

As soluções estoque dos padrões foram preparadas em metanol, 
individualmente, na concentração de $1000 \mathrm{mg} \mathrm{L}^{-1}$. Uma solução contendo os dois padrões a $100 \mathrm{mg} \mathrm{L}^{-1}$ foi preparada também em metanol, a partir da diluição da solução estoque. Posteriormente, foram obtidas as soluções intermediárias e as de trabalho por meio de diluições sucessivas em fase móvel (metanol:água 50:50, v/v). Estas soluções foram utilizadas na determinação da linearidade do detector, nas fortificações das amostras e na obtenção das curvas analíticas.

\section{Extração das amostras fortificadas}

Um volume de $250 \mathrm{~mL}$ de amostra, água mineral natural Minazul, fortificada nas concentrações de 0,$1 ; 0,5$ e $1,0 \mu \mathrm{g} \mathrm{L}^{-1}$ foi extraído com 3 porções de $50 \mathrm{~mL}$ de diclorometano. Em seguida o solvente foi evaporado até cerca de $0,5 \mathrm{~mL}$, utilizando um rotaevaporador (Büchi). O extrato foi seco com sulfato de sódio anidro, transferido quantitativamente para um tubo de ensaio e evaporado sob fluxo lento de nitrogênio. O resíduo foi redissolvido em $1 \mathrm{~mL}$ de água, filtrado em membrana de $0,45 \mu \mathrm{m}$ sendo $100 \mu \mathrm{L}$ analisados por cromatografia líquida de alta eficiência.

\section{Equipamento}

Foi utilizado um cromatógrafo líquido da Agilent, modelo 1100 Series; constituído de uma bomba quaternária, autoamostrador, desgaseificador, e um detector espectrofotométrico de absorção no UV/Vis, de comprimento de onda variável. A coluna utilizada foi da Mallinckrodt Baker, modelo Bakerbond HPLC, C18, 4,6 x 250 $\mathrm{mm}$ x $5 \mu \mathrm{m}$. As condições de operação foram: fase móvel água:metanol (50:50, v/v), eluição isocrática, vazão $1 \mathrm{~mL} \mathrm{~min}{ }^{-1}$, tempo de corrida de 15 minutos e detecção no UV em $247 \mathrm{~nm}$.

A validação do método proposto foi obtida por meio da avaliação dos parâmetros de linearidade, limite de detecção (LOD), limite de quantificação (LOQ), exatidão e precisão.

\section{RESULTADOS E DISCUSSÃO}

Este trabalho descreve um método analítico que está sendo utilizado na determinação dos herbicidas hexazinone e tebutiuron em água subterrânea proveniente do Aqüúfero Guarani. O monitoramento destes princípios ativos em água subterrânea é de grande relevância neste sistema hidrológico onde a vulnerabilidade é alta, e especialmente porque a água subterrânea, nesse caso, é a fonte primária (ou principal) de água potável que abastece a população, particularmente no Estado de São Paulo.

O método é baseado em extração líquido-líquido, que embora trabalhoso é mais barato do que os métodos que usam extração em fase sólida, onde os cartuchos são caros e depende muitas vezes de importação.

A resolução na linha de base dos herbicidas foi obtida, após um estudo intensivo das condições cromatográficas, utilizando uma coluna de fase reversa tipo C-18 e eluição isocrática com uma mistura de metanol/água 50:50, v/v. A Figura 2 mostra os cromatogramas obtidos das amostras branco e fortificada. Pode-se observar que não há picos de interferentes coeluindo nos tempos de retenção dos compostos estudados.

O solvente utilizado na extração foi o diclorometano, pois é um dos mais utilizados devido a capacidade de extrair compostos tendo uma ampla gama de polaridades e é fácil de evaporar, tornando a concentração da amostra rápida.

Após a otimização das condições de extração e cromatográficas, o método foi validado. A validação é necessária para garantir a confiabilidade dos resultados obtidos.
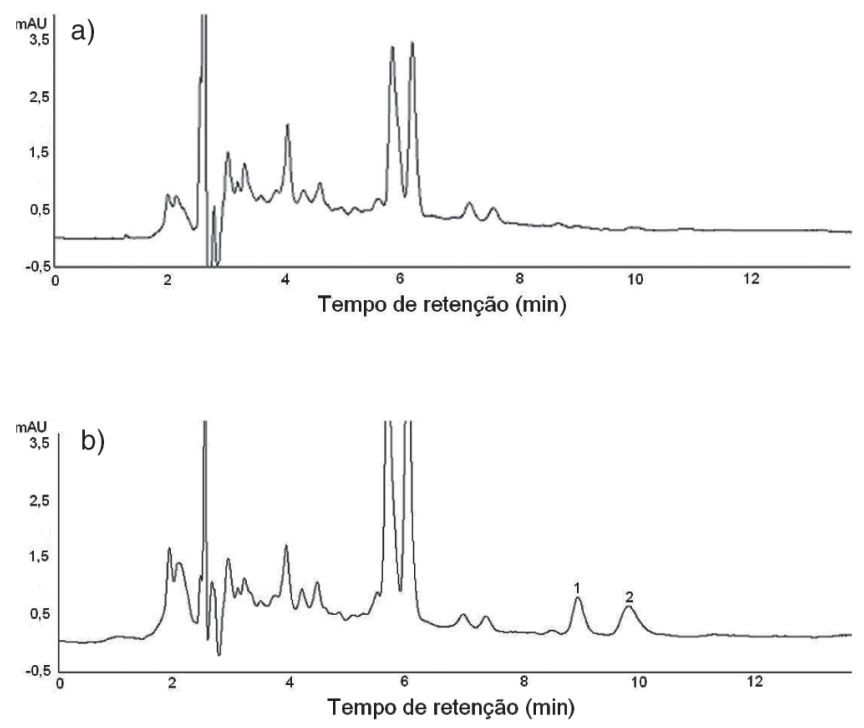

Figura 2. Cromatogramas das amostras: a) branco e b) fortificada inicialmente em $0,1 \mu \mathrm{g} \mathrm{L}^{-1} .1=$ tebutiuron e 2 = hexazinone

Na validação o primeiro e mais simples parâmetro a ser avaliado é a linearidade, que é obtida plotando os valores de área versus a concentração da solução analítica. Realiza-se uma regressão linear pelo método dos mínimos quadrados e verifica-se se o coeficiente de determinação, $\mathrm{r}^{2}$, é adequado. Os desvios da linearidade muitas vezes são difíceis de serem detectados visivelmente, sendo assim sua adequação foi verificada por meio do cálculo dos resíduos entre os valores medidos e os valores calculados a partir da equação de regressão ${ }^{10}$. As curvas analíticas obtidas para o hexazinone e para o tebutiuron mostraram ser lineares na faixa do limite de quantificação do instrumento - $300 \mu \mathrm{g} \mathrm{\textrm {L } ^ { - 1 }}$ pois apresentaram valores de $\mathrm{r}^{2}$ 0,9976 e 0,9987, respectivamente, e os gráficos dos resíduos não demonstraram tendências.

Os limites de detecção (LOD) e os limites de quantificação (LOQ) do instrumento foram calculados utilizando a razão sinal/ ruído de 3 e 10 vezes, respectivamente. Os valores, referentes ao instrumento, do LOD foram 5 e $8 \mu \mathrm{g} \mathrm{L} \mathrm{L}^{-1}$ e LOQ 18 e $22 \mu \mathrm{g} \mathrm{L} \mathrm{L}^{-1}$ para o hexazinone e para o tebutiuron, respectivamente. Os valores, referentes ao método, do LOD foram 0,02 e $0,03 \mu \mathrm{g} \mathrm{L}^{-1}$ e LOQ 0,07 e $0,09 \mu \mathrm{g} \mathrm{L}^{-1}$, para o hexazinone e para o tebutiuron, respectivamente. Estes resultados indicam que o método é suficientemente sensível para detectar a presença dos herbicidas em níveis baixos de concentração. As curvas analíticas estão apresentadas na Tabela 1.

Tabela 1. Curvas analíticas dos herbicidas

\begin{tabular}{lccc}
\hline Herbicida & \multicolumn{3}{c}{ Curvas analíticas } \\
Hexazinone & 2,5198 & $\mathrm{~b}$ & $\mathrm{r}^{2}$ \\
Tebutiuron & 4,2456 & 0,4508 & 0,9976 \\
\hline
\end{tabular}

Nota: $\mathrm{Y}=\mathrm{a}+\mathrm{bx}, \mathrm{a}=$ coeficiente linear, $\mathrm{b}=$ coeficiente angular, $\mathrm{r}^{2}=$ coeficiente de determinação.

A exatidão do método foi determinada por meio da obtenção da \% de recuperação média das amostras fortificadas em triplicatas. Os valores obtidos encontram-se descritos na Tabela 2 e estão dentro da faixa de $70-110 \%$, que é considerada aceitável ${ }^{11}$.

A precisão, na Tabela 2, foi calculada por meio de repetições realizadas no mesmo dia, nas mesmas condições cromatográficas 
Tabela 2. Recuperações e precisões (RSD \%) dos herbicidas hexazinone e tebutiuron $(\mathrm{n}=3)$

\begin{tabular}{lcccc}
\hline Herbicida & $\begin{array}{c}\text { Fortificação } \\
\left(\mu \mathrm{g} \mathrm{L}^{-1}\right)\end{array}$ & $\begin{array}{c}\text { Recuperações } \\
\text { médias }(\%)\end{array}$ & $\begin{array}{c}\text { Repetitividade }^{\mathrm{a}} \\
\text { R.S.D. }(\%)\end{array}$ & $\begin{array}{c}\text { Precisão intermediária }^{\mathrm{b}} \\
\text { R.S.D. }(\%)\end{array}$ \\
\hline Hexazinone & 0,1 & 108,2 & 2,2 & 7,7 \\
& 0,5 & 93,2 & 3,9 & 3,1 \\
Tebutiuron & 1,0 & 90,3 & 3,2 & 3,3 \\
& 0,1 & 101,6 & 3,2 & 5,4 \\
& 0,5 & 90,9 & 1,9 & 5,8 \\
\end{tabular}

$\mathrm{a}=$ mesmo dia , mesmo analista e mesmo equipamento; $\mathrm{b}=$ três dias diferentes, mesmo analista e mesmo equipamento.

e pelo mesmo analista, sendo este tipo de precisão denominado de repetitividade. Também foi avaliada em três dias diferentes, a fim de se determinar a precisão intermediária. Os valores obtidos foram $<8 \%$, indicando que o método está em consonância com a literatura, onde valores $<20 \%$ são considerados aceitáveis ${ }^{11}$.

\section{CONCLUSÃO}

O método proposto mostrou, por meio dos parâmetros de validação, ser eficiente e confiável para a determinação dos herbicidas tebutiuron e hexazinone em amostras de água subterrânea. A extração líquido-líquido apresenta vantagens sobre a extração em fase sólida em relação ao custo, facilidade de obtenção do solvente e, principalmente, quanto à simplicidade, uma vez que utiliza materiais facilmente encontrados em laboratório.

\section{AGRADECIMENTOS}

À Embrapa e FAPESP pelo suporte financeiro do projeto.

\section{REFERÊNCIAS}

1. Roberts, T. R.; Kearney, P. C.; Environmental behaviour of agrochemicals, West Sussex: John Wiley \& Sons Ltd., 1995, p. 328.

2. Gomes, M. A. F.; Spadotto, C. A.; Lanchotte, V. L.; Pesticidas: Revista de Ecotoxicol. e Meio Ambiente 2001, 11, 65.

3. Cerdeira, A. L.; Pessoa, M. C. P. Y.; Bonato, P. S.; Queiroz, R. H. C.; Lanchote, V. L.; Pesticidas: Revista de Ecotoxicol. e Meio Ambiente 2002, 12, 99.

4. Spadotto, C. A.; Gomes, M. A.; Hornsby, A. G.; Pesticidas: Revista de Ecotoxicol. e Meio Ambiente 2002, 12, 1.

5. Kubilius, D. T.; Bushway, R. J.; J. Chromatogr., A 1998, 793, 349.

6. Holt, R.; J. Agric. Food Chem. 1981, 29, 165.

7. Bouchard, D. C.; Lavy, T. L.; J. Chromatogr. 1983, 270, 396.

8. Fischer, J. B.; Michael, J. L.; J. Chromatogr., A 1995, 704, 131.

9. Queiroz, S. C. N. Q.; Melo, L. F. C.; Jardim, I. C. S. F.; J. Chromatogr., A 2002, 948,171.

10. http://www.inmetro.gov.br/kits/doqcgcre008r01.pdf, acessada em Maio 2004.

11. Concil Directive 94/43/EC.; Off. J. Eur. Com. 1994, L227, 31. 